\title{
Photorefractive materials, effects, and devices: control of light and matter
}

\author{
K. Buse • C. Denz • W. Krolikowski
}

Published online: 29 April 2009

(C) Springer-Verlag 2009

In June 2009 about 140 researchers from all over the world will meet for three days in Bad Honnef, Germany, to discuss newest results at the 12th topical meeting "Photorefractive Materials, Effects, and Devices: Control of Light and Matter" (http://www.pr09.de). In order to allow publishing highquality conference contributions in an archival way, this advance feature issue has been realized. We are glad that many researchers took this opportunity, such that an impressive compilation of novel insights into actual material physics, optical effects, and possible applications can be found in this special issue.

Research is per definition not static at all. In particular, the field of photorefraction - which is now progressing for more than 40 years-has undergone impressive changes within the last decade.

Among the many different photorefractive materials that have been studied, lithium niobate is playing a prominent role. Last year, more than 1000 publications appeared worldwide that are based on this very versatile material.

\section{K. Buse $(\bowtie)$}

Institute of Physics, University of Bonn, Wegelerstr. 8,

53115 Bonn, Germany

e-mail: kbuse@uni-bonn.de

Fax: +49-228-734038

\section{Denz}

Institute of Applied Physics, University of Münster, Corrensstr. 2, 48149 Münster, Germany

e-mail: denz@uni-muenster.de

Fax: +49-251-8339811

W. Krolikowski

Laser Physics Centre, Australian National University,

John Carver Building, 58C, Canberra, ACT 0200, Australia

e-mail:wzk111@rsphysse.anu.edu.au

Fax: +61-2-61250029
Sillenite-type crystals, semiconductor crystals, and in particular polymers and nanoparticle-doped hybrid materials are also active fields of research. Driving forces of this work are in many cases interactions of the photorefractive effect with other phenomena, or the ability to learn about material physics by using the photorefractive effect as a method to study for example charge transport properties.

The articles also show that entirely new mechanisms can be discovered by in-depth studies of the light-matter interaction of photorefractive materials. Here we want to highlight the article "Large-scale magnetic field influence on trap recharging waves in InP:Fe and GaAs:Cr", which Prof. Dr. M.P. Petrov, Ioffe Physico Technical Institute, Russian Academy of Sciences, St. Petersburg, Russia, submitted together with his coworkers for publication in this feature issue only a few days before his death in December 2008. It was with great sadness that we learnt that our colleague and friend passed away. We will always commemorate him as a great scientist having a leading role in photorefractive material investigations, who has stimulated a worldwide experimental and theoretical interest in this field. Also, we honor his active and always engaged contribution that he made to the conference series on "Photorefractive Materials, Effects, and Devices."

Another trend has been immensely successful in the past years and can be clearly found throughout this feature issue: nonlinear beam propagation in refractive index structures induced in photorefractive materials results in a number of "hot" topics, e.g., slow light, solitons, photonic lattices, and dynamic waveguides. This attractive field benefits from a key feature of the photorefractive effect: In opposite to Kerr materials, already pretty low light power causes strong nonlinearities allowing to influence light propagation and to couple beams in a very effective manner. 
Photorefractive applications have also undergone an impressive change: In the last century, holographic data storage and optical image processing were research foci. These days we see a strong interest to use photorefractive materials, in particular, in optical metrology: improved Raman filters, advanced optical coherence tomography, extremely sensitive all-optical vibration analysis, and measurements of fluid dynamics are examples of actual highlights of applications that you discover throughout this special issue.

What are the future perspectives? Three main streams seem to be evident by following actual developments:

1. Photonics in general will continue to be of growing relevance for science as well as for industrial applications.

2. Optical materials will be improved further including novel techniques of preparation derived from nanosciences.
3. Lasers, as the enabler of almost all photonic devices, are still becoming cheaper, more efficient, and compact at a high rate supplying all timescales, powers, and frequency ranges that are necessary for future photonics applications.

These are prerequisites that will allow the photorefractive effect to play an important role also in the future.

Finally, we express our gratitude to all persons who made this feature issue possible, in particular Prof. Dr. Frank Träger, Editor in Chief, for his continuous support as well as Ms. Raja Bernard and Ms. Jutta Kaisig for their professional administrative assistance.

Karster Buse Cornelia Denz Wieslaw Krolikowski 